\title{
Re-Engineering Alzheimer Clinical Trials: Global Alzheimer's Platform Network
}

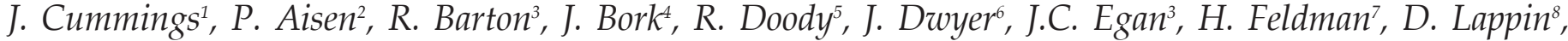 \\ L. Truyen ${ }^{9}$, S. Salloway ${ }^{10}$, R. Sperling 11 , G. Vradenburg ${ }^{4}$ for the GAP-NET Working Groups*
}

* GAP-NET Working Group: Paul Aisen, Russell Barton, Randy Bateman, Jason Bork, Adam Boxer, Mark Brody, William Burke, Jeffrey Cummings, Rachelle Doody, John Dwyer, Johanna Carmel, Howard Feldman, Debra Lappin, Allan Levey, Gad Marshall, Marshall Nash, Dorene Rentz, Craig Ritchie, Stephen Salloway, Lon Schneider, Joy Snider, Reisa Sperling, Pierre Tariot, Luc Truyen, George Vradenburg, Michael Weiner

1. Cleveland Clinic Lou Ruvo Center for Brain Health, Las Vegas, NV, USA; 2. University of Southern California, Los Angeles, CA, USA; 3. Eli Lilly, Indianapolis, IN, USA; 4. Pintail Solutions, Indianapolis, IN, USA; 5. Baylor College of Medicine, Alzheimer's Disease and Memory Disorder Center, Baylor, TX, USA; 6. Global Alzheimer's Platform Foundation, USA; 7. University of British Columbia, Vancouver, BC, USA; 8. FaegreBD Consulting, Washington, DC, USA; 9. Johnson \& Johnson, New Brunswick, NJ, USA; 10. Brown University, Providence, RI, USA; 11. Harvard Medical School, Boston, MA, USA

Corresponding Author: Jeffrey Cummings, MD, ScD, Cleveland Clinic Lou Ruvo Center for Brain Health, 888 W Bonneville Ave, Las Vegas, NV 89106, T: 702.483.6029, F: 702.722.6584, E: cumminj@ccf.org

J Prev Alz Dis 2016;3(2):114-120

Published online March 4, 2016, http:/ / dx.doi.org/10.14283/jpad.2016.93

\begin{abstract}
Alzheimer's disease (AD) drug development is costly, timeconsuming, and inefficient. Trial site functions, trial design, and patient recruitment for trials all require improvement. The Global Alzheimer Platform (GAP) was initiated in response to these challenges. Four GAP work streams evolved in the US to address different trial challenges: 1) registry-to-cohort web-based recruitment; 2) clinical trial site activation and site network construction (GAP-NET); 3) adaptive proof-of-concept clinical trial design; and 4) finance and fund raising. GAP-NET proposes to establish a standardized network of continuously funded trial sites that are highly qualified to perform trials (with established clinical, biomarker, imaging capability; certified raters; sophisticated management system. GAP-NET will conduct trials for academic and biopharma industry partners using standardized instrument versions and administration. Collaboration with the Innovative Medicines Initiative (IMI) European Prevention of Alzheimer's Disease (EPAD) program, the Canadian Consortium on Neurodegeneration in Aging (CCNA) and other similar international initiatives will allow conduct of global trials. GAP-NET aims to increase trial efficiency and quality, decrease trial redundancy, accelerate cohort development and trial recruitment, and decrease trial costs. The value proposition for sites includes stable funding and uniform training and trial execution; the value to trial sponsors is decreased trial costs, reduced time to execute trials, and enhanced data quality. The value for patients and society is the more rapid availability of new treatments for AD.
\end{abstract}

Key words: Global Alzheimer Platform, Alzheimer's disease, clinical trials, recruitment, certification, registry, drug development, drug discovery.

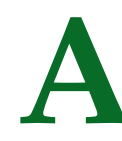

lzheimer's disease $(\mathrm{AD})$ is an increasing threat to public health, becoming more common as the world's population ages. There are currently 35 million individuals affected worldwide and this will grow to 120 million people or more by 2050 (1). Despite the growing need, no new novel agent has been approved for the treatment of AD in over a decade (2).

There is a growing sense of urgency to re-engineer the approach to developing new therapies for AD. The emerging catastrophe of dementia was a major theme of the G8 Summit led by United Kingdom (UK) Prime Minister, David Cameron in December 2012. In Europe the momentum for change led to the Innovative Medicines Initiative's (IMI) European Prevention of $\mathrm{AD}$ (EPAD) program and in the US to an $\mathrm{AD}$ summit organized by the Global Chief Executive Officer (CEO) Initiative on Alzheimer's (CEOi) and the New York Academy of Sciences in November 2013 and subsequent Global Alzheimer's Platform (GAP) design and planning meetings in 2014 (3). Multiple stakeholders including industry leaders, academicians, government officials, non-governmental organizations, advocacy group leaders, and philanthropists participated in the GAP design and planning process. GAP conceptualized a transformative program for $\mathrm{AD}$ drug development and clinical trials addressing many of the issues contributing to the inefficiency, slowness and suboptimal quality of current $\mathrm{AD}$ clinical trials. Four work groups were established: registry-to-cohort (now called TrialReady Cohort for Preclinical and Prodromal AD [TRCPAD]), site activation (now called GAP network [GAPNET], innovative trial design, and GAP financing (3). GAP is intended to deliver consistently high quality performance, enable novel trial designs (ie., adaptive trial designs), and incorporate mechanisms for information and data sharing designed to accelerate scientific learning and clinical translation (3).

AD drug development takes an average of 13 years and costs $\$ 5.6$ billion (including the cost of failures and 
capitalization costs) (4). Phase 3 studies are the longest and most expensive element of the development cycle. The high cost of Phases 2 and 3 reflect in part the nonintegrated nature of the drug development ecosystem and requirement to reconstruct multiple resources to achieve each step of site preparation, trial conduct, and regulatory submission. The absence of an organized $\mathrm{AD}$ clinical trial enterprise increases the time required to recruit for trials and to advance new therapies, increases the costs and duration of trials for sponsors, and delays the availability of treatment for persons in need of therapeutic intervention. The financial costs and risks of $\mathrm{AD}$ drug development decreases the number of agents that can be advanced, discourages some potential sponsors from attempting to develop drugs for $\mathrm{AD}$, and ultimately decreases the availability of new treatments for persons with or at risk for AD.

The purpose of the GAP-NET Working Group is to consider challenges to the conduct of clinical trials, identify solutions, and construct a standing clinical trial network that will conduct clinical trials with efficiency, cost-effectiveness, timeliness and high quality. Here we describe the background, structure, and purpose of the network of clinical trial sites designed to advance this endeavor.

\section{The Alzheimer's Disease Clinical Trial Process is Broken}

The system for implementation, conduct, and monitoring of AD clinical trials is broken (Table 1) $(5,6)$. The compromised state of AD clinical trials contributes to the low success rate of AD drug development both directly by making it difficult to demonstrate a drugplacebo difference and indirectly by discouraging investment in $\mathrm{AD}$ drug development by pharmaceutical and biotechnology companies $(2,7)$.

Recruitment to trials is slow, with some trials recruiting at a rate as low as 0.2 patients per site per month and requiring 1-2 years to recruit patients for 6 month trials. To compensate for slow recruitment, the number of trial sties is increased and inclusion of sites in multiple world regions is common $(8,9)$ Increasing the number of trial sites invites greater inter-rater variability The effects of globalization on trial efficiency have not been thoroughly studied; recent analyses suggest that including many trial sites from multiple regions increases variability in baseline characteristics and in measures of disease course $(10,11)$.

Site rater training is redundant with repetitious training on the same instrument for every trial at every trial site even if training was recently completed by another sponsor. Different sponsors may use slightly different versions of the same instruments requiring the raters at each site to remember these differences, increasing opportunities for errors and protocol deviations $(12,13)$. Rater drift is common, requiring on-going rater monitoring and remedial training (8).

Table 1. Challenges to the optimal conduct of AD clinical trials

Unstable funding adversely affecting site personnel and resources

Lack of appropriate funding for many trial-related administrative costs

Lack of experienced raters

Need to retrain raters for each trial even if recently trained on the same instrument for another trial

Lack of experienced sites and absence of a mechanism for developing new sites

Lack of site infrastructure

- Financial

- IRB

- Pharmacy

- Regulatory

- Technical (MRI, PET, LP)

- Space

Long start-up times (IRB approval, contract approval, budget approval)

Lack of widely available, up-to-date quality information regarding site performance

Slow recruitment

High screen failure rate

Recruitment of patients into AD trials who lack a diagnosis of $\mathrm{AD}$

Low retention rates

Protocol violations

Missing data

Lack of experience with electronic data capture, electronic data entry, and electronic data management

Lack of standard operating procedures

Need to recreate the site network for each trial

AD - Alzheimer's disease; IRB - institutional review board; LP - lumbar puncture; MRI - magnetic resonance imaging; PET -positron emission tomography

There is no standing network for industry trials, and a trials site network must be re-identified and re-constructed for each trial. Contract research organizations (CROs) keep databases of trial site performance but these are not comprehensive, up-todate, or publically available. Trial site availability and trial-related revenue fluctuate. When few trials are available, sites may dismiss staff; when a new trial becomes available, sites must hire and retrain individuals. Experienced staff with valuable expertise may be lost in this cycle. Trial budgeting is pro-rated so start-up costs often must be covered from other funds. Trial budgeting is usually based on visit costs with training, data entry, and administrative costs often uncompensated. Inexperienced sites may be under-budget and fail for 
economic reasons, making it difficult to expand the total number of trial sites.

Administrative procedures such as contract and grant negotiation and budget review are repeated for each trial at each site. Institutional Review Boards (IRBs) at each site review the protocol and make adjustments to the informed consent, creating variability in informed consent at sites across the trial as well as delay in trial site activation and trial initiation.

Because of the boom and bust nature of current $\mathrm{AD}$ trial processes, data collected on trial participants is lost to future trials, participants are not advised of the impact of their personal contributions, and the reasons for trial failure as well as relevant participant data are lost to the field.

The quality and uniformity of patient populations recruited for trials is suboptimal. Recent studies have shown that approximately $20 \%$ of patients diagnosed with $\mathrm{AD}$ dementia and included in trials have no amyloid burden in the brain as determined by amyloid positron emission tomography (PET) $(14,15)$. The absence of amyloidopathy indicates that the diagnosis may be incorrect; the absence of the target pathology will compromise potential efficacy of any anti-amyloid therapies being investigated.

There is currently limited ability to identify amyloidbearing individuals particularly in the preclinical and prodromal stages of AD. Amyloid imaging or cerebrospinal fluid (CSF) amyloid studies are required to identify these study candidates, and there is a high percentage of screen failures. In a recent trial where a rigorous approach was taken to document the presence of pathological CSF AD biomarkers, more than $50 \%$ of subjects with possible prodromal $\mathrm{AD}$ had non-pathologic CSF testing (16). Amyloid imaging is expensive and adds substantially to the total cost of trials. Cerebrospinal fluid measures of amyloid beta-protein $(A \beta)$, total tau and phospo-tau are more accessible and less costly, offering an alternative for biomarker diagnostic support. However there are issues to resolve around the quality and reliability of the current assays (17) as well as the readiness of investigators to enroll patients in this procedure.

\section{GAP-NET}

The lack of a well-funded, well-trained, fast-start, standing network of $\mathrm{AD}$ clinical trial sites is slowing the development of new agents for $\mathrm{AD}$ and contributing to the high cost of $\mathrm{AD}$ drug development. It is this problem that GAP-NET is designed to address. Adequate funding can stabilize sites, enabling them to retain key staff, shorten start-up times, recruit more rapidly, and retain subjects more effectively. Established roles and responsibilities of salaried staff will insure the continuity of site performance in clinical and administrative roles. Pre-identification of sites for the network will eliminate site surveys and shorten the times of trial network construction. Expert financial management systems and standardized master site agreements with sponsors will be established at GAP-NET sites and will enhance administrative efficiency. Appropriate medication storage and accountability will be expected at GAPNET sites. Data collection, entry, transfer systems, and expert use capability will be required of GAP-NET sites. Currently un-funded activities such as data entry and responding to vendor queries will be performed better and more rapidly with proper subsidies. Sites will be monitored for data quality, start-up times, trial conduct, protocol compliance, recruitment rates, and retention of patients in trials. Trial-site metrics will be established and sites failing to perform appropriately will be excused from the network. Development of a comprehensive site performance database will allow continuous remodeling of the network to include the best performing sites, enhance performance of successful sites, and identify site best practices.

Certification of raters will depend on demonstration of skill in administration of all tests relevant to the population appropriate for the GAP-NET trials. Precertification and site qualification will reduce redundancy and decrease the burden on sites. Use of agreed-upon versions of instruments will reduce variability, decrease site demands, and allow greater comparability across trials. Training and certification of new raters will facilitate growth of site teams and expansion of the site network.

Improved site and personnel quality along with greater use of biomarkers will result in improved diagnostic accuracy and more uniform subject characteristics. Seamless access to technical resources such as PET, magnetic resonance imaging (MRI) and lumbar puncture (LP) will be site requirements.

Use of central IRBs can also reduce trial start-up times by decreasing redundancy of reviews at each institution and establishing templates to which trials and reviewers can adhere. Central IRBs such as the reliance model championed by the National Center for Advancing Translational Science (NCATS) will be considered for GAP-NET (18).

Identification and construction of trial-ready cohorts of patients through registries (discussed below) and other outreach mechanisms will abbreviate recruitment times. More aggressive use of traditional and social media can help identify appropriate trial candidates and accelerate trial recruitment.

Enhanced site performance and recruitment will mean that fewer sites will be needed for trials. Smaller sample sizes will be required and variability in data collection and trial conduct will be reduced. Requiring fewer sites and shortened recruitment periods will decrease the cost of trials and allow more drugs to be tested. These advantages represent a value proposition for sponsors, attracting them to work with the network to conduct 
trials and advance therapeutics. The value for those with or at risk of the disease is acceleration of innovative medicines. GAP-NET will be available for both early stage proof-of-concept trials and for pivotal trials and will conduct trials across the spectrum of cognitive normal elderly to prodromal $\mathrm{AD}$ and $\mathrm{AD}$ dementia.

Inclusion of patients in higher quality trials that are better run, better supervised, and lead to better quality data is more ethical and reflects the precious resource that patient participation in clinical trials represents. Recruitment of patients to trials that have little chance of leading to new therapies is at best disrespectful and must be discouraged.

Pre-competitive cooperation by pharmaceutical companies working with academic and other commercial entities is imperative for GAP-NET to succeed. Use of the network will require that the sponsor agree to use the specific form of each instrument that has been selected. Similarly, sponsors must agree that the certification and qualification of the sites is acceptable and need not be repeated for their trial. Common language acceptable to sponsors and institutions hosting GAP-NET sites will need to evolve for budgets, contracts, and IRBs. Sponsors will benefit in terms of cost and time savings and quality enhancement by accepting GAP-NET standards. Innovative thinking within the biopharmaceutical industry is resulting in significantly increased transparency of clinical research and safety information and willingness to consider collaboration on study design, measures of clinical efficacy, and biomarkers (19). This collaborative approach will facilitate achieving GAP-NET objectives. A goal of GAP-NET is to enhance data sharing among stakeholders to facilitate treatment development.

Cooperation of regulatory authorities (Food and Drug Administration [FDA] and European Medicines Agency [EMA]) is critical to the success of GAP-NET. Test procedures, instrument choice, use of run-in data, and data collection and standards require regulatory discussion to assure the acceptability of data collected by GAP-NET for regulatory purposes.

GAP will collaborate with and learn from existing models of national and international site collaboration such as the NCATS, Alzheimer's Disease Cooperative Study (ADCS), TransCelerate, and the European and Developing Countries Clinical Trials Partnership (ADCPT). The Patient Centered Outcomes Research Institute (PCORI) and the National Institutes of Health (NIH) including National Institute of Aging (NIA), NCATS, and NIH-sponsored programs such as the NIH Health Care Systems Research Collaboratory will also have key roles in the success of GAP-NET. The processes adopted by GAP-NET include many initiatives recommended in the Re-Engineering Clinical Trials Initiative of NIH (20-22).

Planned site and network characteristics of GAP-NET are presented in Table 2 .

\section{Table 2. Site and network characteristics of GAP-NET}

\section{Site characteristics}

- Optimized infrastructure

o Staff

o Space

o Electronic resources

- Stable site funding

- Stable team with assigned responsibilities

o FRB and informed consent

o Recruitment

o Rating

o Data management

o Regulatory compliance

o Laboratory

o Imaging

o Pharmacy

o Budgeting and financial monitoring

- Experienced raters

- Certified raters

- Rapid start-up times

- High rate of recruitment

- Access to diverse, representative populations

- High rate of retention

- Technical resources (PET, MRI, LP)

- Standard operating procedures and documentation of all trialrelated procedures

Network characteristics

- Qualified sites with features listed above

- Experienced clinical monitoring team

- Biomarker and biomarker data management capability

- Central IRB

- Expert financial systems

- Master site agreements

- Site management and performance tracking

- Comprehensive site database

- Flexibility to meet needs of trials with variable characteristics

- Regulatory expertise

- Compliant, flexible, reliable data systems for trials

- Media and communications strategy (national, site-specific)

- DSMB for network trials

- Seamless interface of sites with GAP registry and GAP cohort established by TRC-PAD

DSMB - data safety and management board; IRB - institutional review board; LP - lumbar puncture; MRI - magnetic resonance imaging; PET -positron emission tomography; TRC-PAD - trial-ready cohort for prevention of Alzheimer's disease

Sites will be included in GAP-NET with the aim of having enough sites within the network to conduct all clinical trials presented by sponsors. Both academic sites 117 
affiliated with major medical centers and independent non-academic sites will be included in the network. Eleven pilot sites have been identified by GAP in its pilot phase. It is anticipated that there may be up to 100 US sites in the GAP-NET, and these will collaborate with EPAD and Canadian Consortium on Aging and Neurodegeneration (CCNA) sites, as well as other nations' sites meeting the GAP-NET standards, in order to conduct global trials.

\section{Registry-to-Cohort Work Group}

Novel mechanisms are required to speed patient recruitment to trials. Recruitment constitutes the greatest bottleneck for clinical trial conduct in AD and in many other disorders (10). TRC-PAD is an innovative approach using registries to identify potential participants for trials. The Brain Health Registry (BHR) will be central to the process of feeding a central GAP Registry, as will other registries and non-registry based outreach mechanisms such as collaborations with large physician practices and Medicare/Medicaid enrollment lists as well as use of mobile computing.

For the GAP registry, interested individuals will enroll on the web-based BHR or collaborating registry, provide demographic information, complete questionnaires, take online cognitive assessments and contribute genetic or other clinical information available through remote collection methods. Based on these data, adaptive reiterative algorithms will be developed to select participants most likely to meet trial entry criteria (Figure 1). These potential subjects will be referred to GAP-NET sites for biomarker assessment (e.g., amyloid imaging) and further testing. Amyloid imaging will be performed at least in part in conjunction with the Imaging Dementia - Evidence for Amyloid Scanning (IDEAS) Study. Individuals meeting all criteria will comprise the GAP Cohort and will be entered into GAP-NET clinical trials (Figure 2). The algorithm-based approach is hypothesized to increase the number of patients referred to trials, improve the appropriateness of the referred subjects, reduce the screen failure rate, and decrease of cost of screening. GAP-NET sites will receive referrals for trials from the GAP Cohort. The creation of trial-ready-cohorts is proposed as a means of speeding recruitment and shortening trial cycle times. Other registries (endalznow.org; healthybrains.org; sitebased registries) will also be included in the TRC-PAD initiative as channels for referring patients to the GAP registry. Tracking the trajectory of registry and cohort patients after registration and before randomization will provide additional information on drug-induced change in trajectory after trial entry and could help select patients for trials. On-line assessments may reduce the burden on care partners and clinical trial sites to collect participant data.

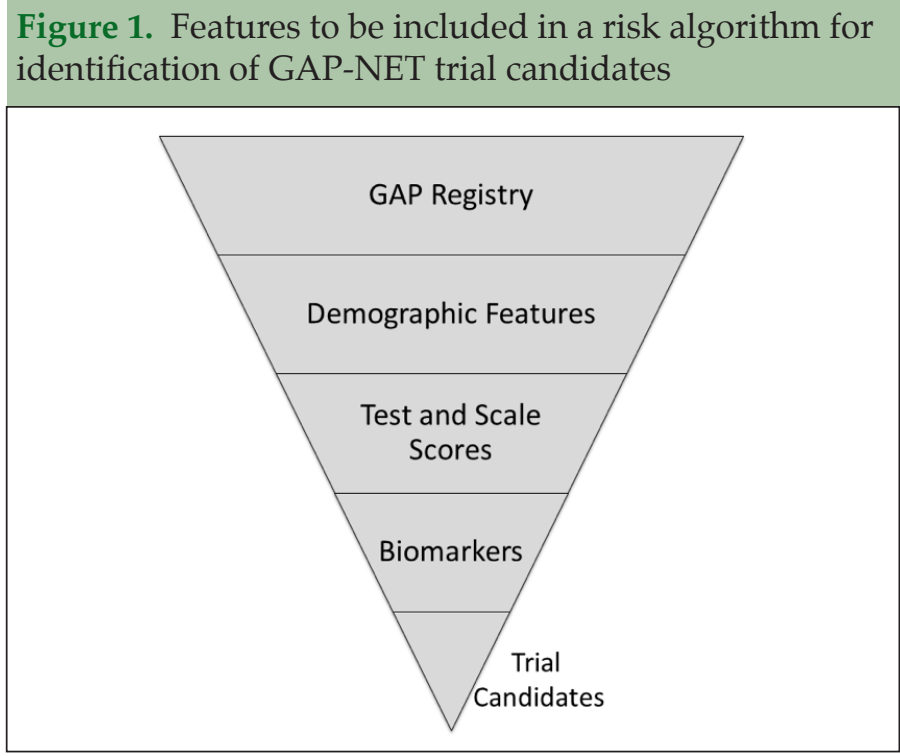

Figure 2. TRC-PAD mechanism for identifying patients for GAP-NET trials (BHR - Brain Health Registry)

$\left.\begin{array}{c}\text { BHR; Other } \\ \text { Channels }\end{array}\right\rangle$ GAP Registry $\sum \begin{gathered}\text { Biomarker } \\ \text { Assessment }\end{gathered} \gg$ GAP Cohort $\sum \begin{gathered}\text { GAP-NET } \\ \text { Sites and } \\ \text { Trials }\end{gathered}$

\section{European Prevention of Alzheimer's Disease and Canadian Centers for Neurodegeneration and Aging Initiative}

The European IMI inaugurated the EPAD project to create a network of trial sites and conduct clinical trials using adaptive designs to test multiple agents (23-27). The 12 Trial Delivery Centers (TDCs) included in the EPAD network will have features similar to those of GAP-NET, and the two networks will collaborate to allow conduct of trials using sites in both the US and Western Europe. Similarly, the CCNA is collaborating with GAPNET to allow inclusion of Canadian sites in the execution of multi-regional trials.

Chinese, Japanese and South American site leaders are engaged in GAP-NET discussions. EPAD is focused on prevention trials in patients with preclinical or prodromal AD; GAP-NET plans to conduct Phase 2 and 3 trials in all stages of $\mathrm{AD}$.

\section{GAP-NET and Drug Development}

GAP-NET cannot lead to new treatments without a concomitant improvement in AD drug discovery and delivery of a pipeline of high-quality pharmaceutical agents capable of impacting AD pathology. GAP-NET can test drugs more quickly and can provide better data that will allow sponsors to more rapidly decide whether or not to advance a compound for further testing. GAPNET can assist in seeing that effective agents have a 
"quick win" in proof-of-concept trials and that ineffective agents "fail fast", reducing the investment in drugs that cannot succeed (28). GAP-NET will not increase the AD drug development success rate without having highly efficacious agents to test in trials. Discovery of better treatments depends on deepening our understanding of the basic biology of $\mathrm{AD}$, comprehending the cellular mechanisms of neurodegeneration, and distinguishing normal and abnormal aging. GAP-NET is an engine that needs to be fueled by optimized compounds and combinations of agents developed in academic, pharmaceutical and biotechnology laboratories that meaningfully impact the biology of AD. Investment in $\mathrm{AD}$ drug discovery is a key element in resolving the crisis posed by $\mathrm{AD}$ and will complement the transformative trial solution presented by GAP-NET.

\section{Summary}

GAP-NET intends no less than the re-engineering of AD clinical trials as currently conducted to a standing, structured, integrated, quality system capable of recruiting patients and efficiently escorting them through trials up to two years faster than today's standard. GAPNET represents a disruptive transformation that will have effects throughout the AD drug development ecosystem; lessons learned from GAP-NET may influence organization of trials in other neurodegenerative disorders and other disease states (22). The GAP-NET will be expanded from the 11 sites in the pilot phase to the number of sites needed to enter patients and conduct trials in a timely way. The total number of necessary sites will be reduced compared to current standards by enhanced recruitment, concentration of registry activity around GAP-NET sites, decreased data "noise", and reduced screen fails. Sites meeting quality criteria will be GAP-NET partners regardless of their academic or private nature; poor performing sites will be excused from the network. An evolving clinical trial database will allow the investigation and publication of trial site best practices. Structured introduction of new instruments such as patient- and caregiver-reported outcomes can be facilitated and systematically planned in the network and included in trials after regulatory review by FDA and EMA. GAP-NET will develop capacity for site qualification (clinical, biomarker, imaging), rater certification, site monitoring, and growth of the number of available sites. GAP-NET will be prepared to collaborate with IMI-EPAD, CCNA and other quality networks meeting GAP-NET standards to conduct trials worldwide. GAP-NET together with a robust AD pipeline can deliver new treatments to patients faster.

Disclosures: Paul Aisen has served as a consultant to the following companies: NeuroPhage, Elan, Eisai, Bristol-Myers Squibb, Eli Lilly, Merck, Roche, Amgen, Genentech, Abbott, Pfizer, Novartis, AstraZeneca, Janssen, Medivation, Ichor, Lundbeck, Biogen, iPerian, Probiodrug, Anavex, Abbvie, Janssen, Cohbar. Dr. Aisen receives research support from Eli Lilly, the Alzheimer's Association and the NIH [NIA U01-AG10483 (PI), NIA U01-AG024904 (Coordinating Center Director), NIA R01-AG030048 (PI), and R01-AG16381 (Co-I)]. Russell Barton is an employee of Eli Lilly.

Jason Bork is a Principle at Pintail Solutions and serves as a project management consultant to Global Alzheimer's Platform Foundation.

Jeffrey Cummings has received in kind research support from Avid Radiopharmaceuticals and Teva Pharmaceuticals. He has provided consultation to AbbVie, Acadia, ADAMAS, Alzheon, Anavex, AstraZeneca, Avanir, BiogenIdec, Biotie, Boehinger-Ingelheim, Chase, Eisai, Forum, Genentech, Intracellular Therapies, Lilly, Lundbeck, Merck, Neurotrope, Novartis, Nutricia, Otsuka, Pfizer, Prana, QR Pharma, Resverlogix, Roche, Suven, Takeda, and Toyoma companies. He has provided consultation to GE Healthcare and MedAvante and owns stock in ADAMAS, Prana, Sonexa, MedAvante, Neurotrax, and Neurokos. Dr. Cummings owns the copyright of the Neuropsychiatric Inventory.

Rachelle Doody provides consultation to AC Immune, Axovant, AZ Therapies, Biogen, Cerespir, Forum, Genentech, Hoffman-LaRoche, Shanghai Green Valley, Riovant, Suven, Transition, Takeda, VTV companies. She has research support (clinical trials) with Accera, Avanir, Genentech, Lilly, Merck, NIH/Sanofi, Pfizer, and Takeda. Other - Hoffman LaRoche (DSMB), Lilly/UCSD (ADCS-DAPC). Lastly, Dr. Doody has stock options with AZ Therapies, QR Pharma, Sonexa, and Transition.

John Dwyer is President and Founding Board Member of Global Alzheimer's Platform and is Chairman of Telcare, Inc.

Johanna Egan is an employee of Eli Lilly.

Howard Feldman has provided consultation through University of British Columbia service agreements with Eli Lilly, Merck, Arena, GE HealthCare, Kyowa Hakko Kirin, Biogen Idec, ISIS Pharmaceuticals from 2012 to present. Dr. Feldman has served on safety monitoring or diagnostic monitoring boards for Eisai and Genentech and has received research support / performed clinical trials sponsored by Pfizer, Eisai, Novartis, Janssen, Lundbeck, Genentech, Roche, and Astra Zeneca. Between 2009-2011, he served as full-time employee of BristolMyers Squibb while on leave from University of British Columbia.

Debra Lappin is a Principal with FaegreBD Consulting. In this capacity, she provides consultation to the Global Alzheimer's Platform Foundation and GAP-Net. FaegreBD Consulting provides consulting services to a wide range of pharmaceutical companies.

Steven Salloway has received grant support from the GAP-NET Foundation which is directly related to the content of this manuscript. He reports grant support and consultation fees from Biogen, Merck, Roche, Genentech, and Lilly, and grant support from Avid and Functional Neuromodulation.

Reisa Sperling has served as a consultant for Merck, Eisai, Janssen, BoehringerIngelheim, Isis, Lundbeck, Roche, and Genentech.

Luc Truyen is an employee of Johnson and Johnson.

George Vradenburg is Founder and Chair, USAgainstAlzheimer's; Convener, The Global CEO Initiative on Alzheimer's.

\section{References}

1. Alzheimer's Disease International, Prince M, Guerchet M, Prina M. Policy Brief for Heads of Government: The Global Impact of Dementia 2013-2050. London: Alzheimer's Disease International, 2013.

2. Cummings JL, Morstorf T, Zhong K. Alzheimer's disease drug-development pipeline: Few candidates, frequent failures. Alzheimers Res Ther 2014;6:37.

3. The New York Academy of Sciences, The Global CEO Initiative on Alzheimer's Disease. Alzheimers Disease Summit: The Path to 2025 Summary and Strategy Report. New York, NY: The New York Academy of Sciences; 2013 Dec 9.

4. Scott TJ, O'Connor AC, Link AN, Beaulieu TJ. Economic analysis of opportunities to accelerate Alzheimer's disease research and development. Ann N Y Acad Sci 2014;1313:17-34.

5. Becker RE, Greig NH. Increasing the success rate for Alzheimer's disease drug discovery and development. Expert Opin Drug Discov 2012;7:367-370.

6. Becker RE, Greig NH. Why so few drugs for Alzheimer's disease? Are methods failing drugs? Curr Alzheimer Res 2010;7:642-651.

7. Hyman BT, Sorger P. Failure analysis of clinical trials to test the amyloid hypothesis. Ann Neurol 2014;76:159-161.

8. Doody RS, Cole PE, Miller DS, et al. Global issues in drug development for Alzheimer's disease. Alzheimers Dement 2011;7:197-207.

9. Cummings J, Reynders R, Zhong K. Globalization of Alzheimer's disease clinical trials. Alzheimers Res Ther 2011;3:24.

10. Grill JD, Galvin JE. Facilitating Alzheimer disease research recruitment. Alzheimer Dis Assoc Disord 2014;28:1-8.

11. Henley DB, Dowsett SA, Chen YF, et al. Alzheimer's disease progression by geographical region in a clinical trial setting. Alzheimers Res Ther 2015;7:43.

12. Connor DJ, Sabbagh MN. Administration and scoring variance on the ADASCog. J Alzheimers Dis 2008;15:461-464.

13. Connor DJ, Sabbagh MN, Cummings JL. Comment on administration and scoring of the Neuropsychiatric Inventory in clinical trials. Alzheimers Dement 2008;4:390-394.

14. Doody RS, Thomas RG, Farlow M, et al. Phase 3 trials of solanezumab for 
mild-to-moderate Alzheimer's disease. N Engl J Med 2014;370:311-321.

15. Salloway S, Sperling R, Fox NC, et al. Two phase 3 trials of bapineuzumab in mild-to-moderate Alzheimer's disease. N Engl J Med 2014;370:322-333.

16. Coric V, Salloway S, van Dyck CH, et al. Targeting Prodromal Alzheimer Disease With Avagacestat: A Randomized Clinical Trial. JAMA Neurol 2015;72:1324-1333.

17. Mattsson N, Andreasson U, Persson A, et al. The Alzheimer's Association external quality control program for cerebrospinal fluid biomarkers. Alzheimers Dement 2011;7:386-395.

18. Kaufmann P, O'Rourke P. Central institutional review board for an academic trial network. Acad Med 2015;90:321-323.

19. The New York Academy of Sciences, The Global CEO Initiative on Alzheimer's Disease. Global Alzheimer's Platform. Aligning resources to drive quality, efficiency and innovation in Alzheimer's clinical trials. Prospectus. New York, NY: The New York Academy of Sciences; 2015 Mar.

20. Zerhouni E. Medicine. The NIH Roadmap. Science 2003;302:63-72.

21. Zerhouni EA. US biomedical research: basic, translational, and clinical sciences. JAMA 2005;294:1352-1358.

22. Institute of Medicine. Envisioning a Transformed Clinical Trials Enterprise in the United States: Establishing an Agenda for 2020: Workshop Summary.
Washington, DC: The National Academies Press, 2012.

23. Alexander BM, Wen PY, Trippa L, et al. Biomarker-based adaptive trials for patients with glioblastoma--lessons from I-SPY 2. Neuro Oncol 2013;15:972978.

24. Lenz RA, Pritchett YL, Berry SM, et al. Adaptive, dose-finding phase 2 trial evaluating the safety and efficacy of ABT-089 in mild to moderate Alzheimer disease. Alzheimer Dis Assoc Disord 2015;29:192-199.

25. Ritchie CW, Molinuevo JL, Truyen L, et al. Development of interventions for the secondary prevention of Alzheimer's dementia: the European Prevention of Alzheimer's Dementia (EPAD) project. Lancet Psychiatry 2015.

26. Vellas B, Carrillo MC, Sampaio C, et al. Designing drug trials for Alzheimer's disease: what we have learned from the release of the phase III antibody trials: a report from the EU/US/CTAD Task Force. Alzheimers Dement 2013;9:438-444.

27. Wason JM, Trippa L. A comparison of Bayesian adaptive randomization and multi-stage designs for multi-arm clinical trials. Stat Med 2014;33:2206-2221.

28. Paul SM, Mytelka DS, Dunwiddie CT, et al. How to improve R\&D productivity: the pharmaceutical industry's grand challenge. Nat Rev Drug Discov 2010;9:203-214. 